\title{
Cross-Cultural Validity, Reliability, and Psychometric Properties of the Persian Version of the Scales for Outcomes in Parkinson's Disease-Psychosocial Questionnaire
}

\author{
Seyed-Mohammad Fereshtehnejad, ${ }^{1,2}$ Farzaneh Farhadi, ${ }^{3}$ Hasti Hadizadeh, ${ }^{3}$ \\ Gholam Ali Shahidi, ${ }^{4}$ Ahmad Delbari, ${ }^{1,5}$ and Johan Lökk ${ }^{1,6}$ \\ ${ }^{1}$ Division of Clinical Geriatrics, Department of Neurobiology, Care Sciences, and Society (NVS), Karolinska Institutet, 14186 Stockholm, \\ Sweden \\ ${ }^{2}$ Firoozgar Clinical Research Development Center (FCRDC), Firoozgar Hospital, Iran University of Medical Sciences, Tehran 15937- \\ 48711, Iran \\ ${ }^{3}$ Medical Student Research Committee (MSRC), Faculty of Medicine, Iran University of Medical Sciences, Tehran 14496-14535, Iran \\ ${ }^{4}$ Movement Disorders Clinic, Department of Neurology, Faculty of Medicine, Iran University of Medical Sciences, Tehran 14496-14535, \\ Iran \\ ${ }^{5}$ Iranian Research Center on Aging, University of Social Welfare and Rehabilitation, Tehran 19857-13834, Iran \\ ${ }^{6}$ Department of Geriatric Medicine, Karolinska University Hospital, 14186 Stockholm, Sweden
}

Correspondence should be addressed to Seyed-Mohammad Fereshtehnejad; sm.fereshtehnejad@ki.se

Received 28 January 2014; Accepted 12 March 2014; Published 7 April 2014

Academic Editor: Jeff Bronstein

Copyright (C) 2014 Seyed-Mohammad Fereshtehnejad et al. This is an open access article distributed under the Creative Commons Attribution License, which permits unrestricted use, distribution, and reproduction in any medium, provided the original work is properly cited.

\begin{abstract}
Objectives. Considering the influence of different motor and nonmotor features of Parkinson's disease (PD), it is important to evaluate the psychosocial functioning of the patients. For this purpose, the scales for outcomes in Parkinson's disease-psychosocial questionnaire (SCOPA-PS) has been previously designed. The aim of our study was to assess the cross-cultural validation and psychometric properties of the Persian version of the SCOPA-PS. Methods. One hundred and ten nondemented idiopathic Parkinson's disease (IPD) patients were consecutively recruited from an outpatient referral movement disorder clinic. Eligible patients filled up a number of questionnaires including the Persian version of SCOPA-PS during the face-to-face interview session and clinical examination to measure disease severity, nonmotor psychiatric symptoms, and health-related quality of life (HRQoL). Results. The highest and lowest correlation coefficients of internal consistency were reported for item 7 on "asking for help" $(r=0.765)$ and item 5 on "sexual problems" $(r=0.553)$. Cronbach's alpha reliability coefficient of the entire scale was $0.87(95 \%$ CI: 0.83-0.90). The Hoehn and Yahr stage $(r=0.34, P<0.001)$, Schwab and England ADL scale $(r=-0.55, P<0.001)$, anxiety $(r=0.64, P<0.001)$, depression $(r=0.71, P<0.001)$, and fatigue $(r=0.35, P<0.001)$ were significantly correlated with the total score of the SCOPA-PS questionnaire. Conclusions. The Persian version of SCOPA-PS is a highly reliable and valid scale to measure psychosocial functioning in IPD patients with different sex, age-group, and educational level, which could be applied in future researches. Disease severity scales, depression, anxiety, fatigue, and different domains of HRQoL were all associated with psychosocial functioning in PD patients.
\end{abstract}

\section{Background}

Parkinson's disease (PD) affects about $2 \%$ of population aged 65 years and over $[1,2]$. In addition to motor symptoms, the disease also represents nonmotor features such as depression, apathy, mood changes, and increasing dependence on others for everyday activities [3]. As a result, it considerably influences the life and psychosocial functioning of PD patients; however, this consequence is rarely evaluated separately and in-depth $[4,5]$. Psychosocial consequences of PD are commonly assessed by means of the health-related quality of life (HRQoL) instruments [6]. 
Since 5 billion of the world population live in the developing countries where life expectancy has been recently increased, these regions have to care for a larger number of patients with PD $[2,7]$. While there are rare evidences on HRQoL and psychosocial aspects of PD available in the developing countries especially those of Asia and Africa, most studies have been performed in the developed countries [813].

Scales for outcomes in Parkinson's disease-psychosocial questionnaire (SCOPA-PS) is an 11-item instrument designed to evaluate psychosocial functioning and severity of mentioned problems in PD patients [14]. It has been validated in few countries and non-English languages including German, Spanish, and Brazilian $[8,14-16]$. The aim of the present study was to perform an independent and cross-cultural validation of the Persian version of the SCOPA-PS. Moreover, we evaluated the psychometric properties of this questionnaire to show the relationships between motor and nonmotor symptoms of PD and psychosocial burden of the disease.

\section{Methods}

2.1. Study Setting. A total number of 110 idiopathic Parkinson's disease (IPD) patients who were consecutively admitted at an outpatient referral movement disorder clinic in Tehran, Iran, between October 2011 and September 2012 were included in the study. This study was a collaborative project between Karolinska Institute, Stockholm, Sweden, and Iran University of Medical Sciences (IUMS), Tehran, Iran. Using a cross-sectional design, eligible participants filled up the SCOPA-PS questionnaire during the face-to-face interview session and clinical examination was also performed together with assessments for psychiatric features, fatigue, and quality of life.

2.2. Ethical Considerations. The study protocol was approved by the Ethics Committee of the Neurology Department at Firoozgar Clinical Research Development Center (FCRDC) (affiliated to Iran University of Medical Sciences) in Tehran, Iran. The completion of the questionnaire was voluntary, and the aims and objectives of the study were explained to each patient before participation.

2.3. Sample and Procedure. Inclusion criteria were diagnosis of IPD based on the United Kingdom (UK) Brain Bank Criteria and age of 35 years or older [17]. Exclusion criteria were moderate-to-severe dementia with the Mini-Mental State Examination (MMSE) of <24 [18] and any patient with atypical Parkinsonian syndromes. After enrollment, a complete clinical examination was done by one neurologist specialized in movement disorders for all eligible participants.

Data collection was performed using four questionnaires including the SCOPA-PS, Parkinson's disease quality of life (PDQ-39), Hospital Anxiety and Depression Scale (HADS), and fatigue severity scale (FSS) to assess various aspects of the disease during the interview session and clinical examination. Demographic information consisted of baseline variables, educational status, and comorbidities. PD-related characteristics including disease duration (time passed from diagnosis), measures of disease severity such as Hoehn and Yahr stage [19], and Schwab and England activity of daily living (ADL) scale were recorded.

The Hoehn and Yahr staging [19] is a widely used clinical rating scale, which evaluates the severity of PD based on motor functional disability and clinical findings consisting of 5 stages. Stage 0 indicates no visible symptoms of PD, and stage 5 shows symptoms on both sides of the body indicating the PD patients who are unable to walk. Therefore, a higher stage shows greater levels of functional disability [19]. The Schwab and England scale is another global scoring system for assessing a PD patient's ability to perform daily activities in terms of speed and independence through a percentage figure, where $100 \%$ indicates total independence and $0 \%$ indicates a state of complete dependence in bed-ridden individuals. Therefore, higher scores show greater level of independence. Moreover, the validated Persian version of the fatigue severity scale (FSS-Per) [20] was used to measure fatigue in recruited PD patients. It contains nine questions asking participants to rate the level of fatigue during the past week, while rating scores range from 1 to 7 for each statement. A total score is obtained as the average of all the item-specific scores where higher scores show more severe fatigue [21]. As a screening tool designed to assess the levels of anxiety and depression in patients attending medical clinics, the Persian version of the Hospital Anxiety and Depression Scale (HADS) was also used [22]. It consists of 14 questions divided into two sections; seven questions are related to anxiety and the other seven questions focus on depression. Each section is worth 0-21 points, providing separate scores for either anxiety or depression where the higher scores demonstrate worse condition [23]. As the most common instrument to measure health-related quality of life in PD patients, we also used Parkinson's disease quality of life questionnaire (PDQ-39) [24]. It contains 39 items assessing eight domains of quality of life (QoL) in PD patients: mobility, activities of daily living (ADL), emotional well-being, stigma, social support, cognitions, communication, and bodily discomfort. All questions in PDQ-39 are coded in a Likert scale from 0 to 4 , where 0 = never, $1=$ occasionally, $2=$ sometimes, $3=$ often, and $4=$ always. The maximum score of 100 on the PDQ39 scale represents the worst conditions, while a zero score represents the best condition of QoL in PD patients [24]. In this study, we used a Persian-translated version of the PDQ39 questionnaire, which has been previously validated [25]. All the assessments were done when the patients were in the "On" state.

2.4. SCOPA-PS Questionnaire. The SCOPA-PS questionnaire [14] is a self-administered 11-item scale assessing psychosocial functioning and the severity of a particular problem during the last month. Items are scored with a four-point Likert scale, from 0 (not at all) to 3 (very much). By adding the scores on the individual items, the sum score is calculated, and by transforming the mentioned sum score into percentage values (on the maximum possible score, 33 points), summary 
index (SCOPA-PS SI) is computed. The higher the summary index is, the worse HRQoL is expected in psychosocial aspects [14].

2.5. Translation and Back-Translation. Three native Persian speakers fluent in English translated the SCOPA-PS into Persian. Later on, an English native fluent in Persian who was not involved in the study back-translated the SCOPAPS into English with no access to the original version of the questionnaire. In the comparison of the first backtranslation with the original version, modifications were made to eliminate all discrepancies. The final joint translation was named SCOPA-PS, Persian version.

\subsection{Statistical Analysis}

2.6.1. Description. Data were analyzed by SPSS software version 17.0 (Chicago, IL, USA). To describe continuous variables, mean [standard deviation (SD)] was calculated and frequency (percentage) was used for nominal and categorical variables. The minimum, maximum, and coefficient of variation (CV) were also reported for each of the items in SCOPAPS questionnaire. In order to guarantee the acceptability of a scale, floor and ceiling effects were calculated, which should be less than $15 \%$ [26].

2.6.2. Reliability. In reliability analysis, internal consistency was assessed using Spearman correlation statistic where the mean score of each item was correlated with the sum of the SCOPA-PS score in Parkinsonian patients. Furthermore, Cronbach's alpha intraclass coefficient and the 95\% confidence interval (CI) of the point estimations were also calculated for the whole questionnaire and within different subgroups of IPD patients regarding age-group, sex, and level of education.

2.6.3. Validity. Spearman correlation was used to evaluate the convergent validity of the total score of the SCOPAPS questionnaire in association with demographics and PDrelated variables.

In all analytical procedures, a two-sided $P$ value $<0.05$ was considered as the statistical significant level to reject the beyond $\mathrm{H} 0$ hypothesis.

\section{Results}

3.1. Sociodemographic Characteristics. The study population consisted of 34 (30.9\%) females and $76(69.1 \%)$ males with the mean age of $61.61(S D=10.97)$ years. Sociodemographic characteristics of the recruited participants are summarized in Table 1. The mean duration of PD was $6.34(\mathrm{SD}=4.74)$ years and the median stage of disease severity was 2 based on the Hoehn and Yahr grading. Depression $(22.7 \%, n=25)$ and hypertension $(16.4 \%, n=18)$ were reported to be the most common comorbidities in PD patients, respectively.

3.2. SCOPA-PS Data Description. The Persian version of the SCOPA-PS was filled up by 110 PD patients with no missing
TABLE 1: Sociodemographic characteristics of Parkinson's disease patients $(n=110)$.

\begin{tabular}{lc}
\hline Characteristics & Value \\
\hline Age (yr) & $61.6(11.0)$ \\
$\quad$ Mean (SD) & \\
Gender number (\%) & $34(31)$ \\
$\quad$ Female & $76(69)$ \\
$\quad$ Male & \\
Level of education number (\%) & $8(7)$ \\
$\quad$ Illiterate & $28(26)$ \\
$\quad$ Primary and/or secondary & $30(28)$ \\
$\quad$ High school/diploma & $43(39)$ \\
$\quad$ College and/or university & $6.3(4.7)$ \\
Duration of disease (yr) & \\
$\quad$ Mean (SD) & $25(23)$ \\
Comorbidities number (\%) & $18(16)$ \\
$\quad$ Depression & $17(16)$ \\
Hypertension & $12(11)$ \\
Cardiovascular disease & $11(10)$ \\
Osteoarthritis & $1.9(0.9)$ \\
Diabetes & \\
Hoehn and Yahr stage & $82.0(16.5)$ \\
Mean (SD) & \\
Schwab and England activities of daily living scale \\
(\%) Mean (SD)
\end{tabular}

value for any of the 11 items. Table 2 describes the distribution of the answers for each question and their mean scores. The highest score was observed in item 11 on "future concerns" with the mean of $1.42(\mathrm{SD}=1.10)$, whereas the lowest score was recorded for item 4 on "significant others" with the mean of $0.38(\mathrm{SD}=0.78)$. The mean relative score of the whole questionnaire was $25.23 \%(\mathrm{SD}=22.20)$ ranging between 0 and $97 \%$. SCOPA-PS had no floor effect, and the ceiling effect was $14.5 \%$.

3.3. Reliability. As listed in Table 2 , all of the items showed significant correlation to the total score of the SCOPA-PS questionnaire (all $P<0.001$ ). The highest and lowest Spearman correlation coefficients were reported for item 7 on "asking for help" (Rho $=0.765)$ and item 5 on "sexual problems" (Rho $=0.553)$. Cronbach's alpha coefficient was also calculated for the assessment of the internal consistency reliability of the questionnaire, which resulted in 0.87 (95\% CI: $0.83-0.90$ ). Deletion of none of the questions would increase Cronbach's alpha coefficient above the formerly calculated overall coefficient (0.869). The highest single-item sensitivity of Cronbach's alpha reliability was reported for item 8 on "loneliness" and item 1 on "difficulty at work" where the coefficient would decrease to as low as 0.850 if either of these two questions was deleted.

Cronbach's alpha reliability coefficient was also calculated within different subgroups of PD patients with regard to age-group, sex, and educational level. As shown in Table 3, 
TABLE 2: Data distribution, description, and Spearman correlation for internal consistency of the SCOPA-PS scores in Iranian Parkinson's disease patients $(n=110)$.

\begin{tabular}{|c|c|c|c|c|c|c|c|}
\hline \multirow{2}{*}{ Item } & \multicolumn{4}{|c|}{ Answer distribution number (\%) } & \multicolumn{2}{|c|}{ Descriptive indexes } & \multirow{2}{*}{ Spearman Rho* } \\
\hline & Not at all $(0)$ & A little (1) & Quite a bit (2) & Very much (3) & Mean & SD & \\
\hline $\begin{array}{l}\text { Item } 1 \\
\text { Work }\end{array}$ & $68 \%$ & $12 \%$ & $12 \%$ & $8 \%$ & 0.60 & 0.99 & 0.656 \\
\hline $\begin{array}{l}\text { Item } 2 \\
\text { Hobbies }\end{array}$ & $65 \%$ & $11 \%$ & $18 \%$ & $6 \%$ & 0.66 & 0.99 & 0.623 \\
\hline $\begin{array}{l}\text { Item } 3 \\
\text { Interacting with others }\end{array}$ & $73 \%$ & $9 \%$ & $14 \%$ & $4 \%$ & 0.47 & 0.86 & 0.601 \\
\hline $\begin{array}{l}\text { Item } 4 \\
\text { Significant others }\end{array}$ & $78 \%$ & $7 \%$ & $13 \%$ & $2 \%$ & 0.38 & 0.78 & 0.596 \\
\hline $\begin{array}{l}\text { Item } 5 \\
\text { Sexuality }\end{array}$ & $64 \%$ & $12 \%$ & $16 \%$ & $8 \%$ & 0.69 & 1.02 & 0.553 \\
\hline $\begin{array}{l}\text { Item } 6 \\
\text { Staying at home }\end{array}$ & $56 \%$ & $10 \%$ & $19 \%$ & $15 \%$ & 0.95 & 1.17 & 0.703 \\
\hline $\begin{array}{l}\text { Item } 7 \\
\text { Help }\end{array}$ & $52 \%$ & $10 \%$ & $22 \%$ & $16 \%$ & 1.03 & 1.18 & 0.765 \\
\hline $\begin{array}{l}\text { Item } 8 \\
\text { Lonely }\end{array}$ & $58 \%$ & $16 \%$ & $19 \%$ & $7 \%$ & 0.75 & 1.01 & 0.740 \\
\hline $\begin{array}{l}\text { Item } 9 \\
\text { Conversation }\end{array}$ & $67 \%$ & $13 \%$ & $17 \%$ & $3 \%$ & 0.55 & 0.87 & 0.588 \\
\hline $\begin{array}{l}\text { Item } 10 \\
\text { Embarrassment }\end{array}$ & $57 \%$ & $15 \%$ & $17 \%$ & $11 \%$ & 0.82 & 1.08 & 0.578 \\
\hline $\begin{array}{l}\text { Item } 11 \\
\text { Future }\end{array}$ & $29 \%$ & $19 \%$ & $33 \%$ & $19 \%$ & 1.42 & 1.10 & 0.565 \\
\hline Total score (crude) & - & - & - & - & 8.33 & 7.33 & - \\
\hline Total score (relative \%) & - & - & - & - & 25.23 & 22.20 & - \\
\hline
\end{tabular}

${ }^{*}$ All correlation coefficients are statistically significant with $P<0.001$.

TABLE 3: Reliability (Cronbach's $\alpha$ ) of the SCOPA-PS questionnaire within various subgroups of Iranian Parkinson's disease patients $(n=$ 110).

\begin{tabular}{lc}
\hline Subgroups & $\begin{array}{c}\text { Cronbach's } \alpha \\
\text { coefficient }(95 \% \mathrm{CI})\end{array}$ \\
\hline $\begin{array}{l}\text { Total }(n=110) \\
\text { Age-group }\end{array}$ & $\mathbf{0 . 8 7}(\mathbf{0 . 8 3}$ to $\mathbf{0 . 9 0})$ \\
$\quad<65 \mathrm{yr}(n=65)$ & $0.88(0.84$ to 0.92$)$ \\
$\quad \geq 65 \mathrm{yr}(n=45)$ & $0.84(0.76$ to 0.90$)$ \\
Gender & \\
$\quad$ Female $(n=34)$ & $0.80(0.68$ to 0.89$)$ \\
$\quad$ Male $(n=76)$ & $0.89(0.85$ to 0.92$)$ \\
Educational level & \\
$\quad \begin{array}{l}\text { Illiterate/primary/secondary }(n=36) \\
\quad \text { College/university }(n=73)\end{array}$ & $0.91(0.85$ to 0.95$)$ \\
\hline
\end{tabular}

the Persian version of the SCOPA-PS questionnaire had higher Cronbach's alpha coefficient in males [0.89 (95\% CI: 0.85-0.92) versus 0.80 (95\% CI: $0.68-0.89)$ ] and less educated participants [0.90 (95\% CI: $0.85-0.94)$ versus 0.83 (95\% CI: $0.76-0.88)]$.
3.4. Validity. Higher Hoehn and Yahr stage (Spearman Rho $=0.34, P<0.001)$ and lower Schwab and England ADL scale (Spearman Rho $=-0.55, P<0.001$ ) were significantly correlated with the total score of the SCOPA-PS questionnaire (Table 4). All of the domains of PDQ-39 were also directly correlated with the SCOPA-PS score and the highest correlation coefficient was recorded in the mobility domain (Spearman Rho $=0.72, P<0.001$ ). The entire score of the PDQ-39 was strongly correlated with the SCOPA-PS score as well (Spearman Rho $=0.82, P<0.001$ ). Moreover, anxiety (Spearman Rho $=0.64, P<0.001$ ), depression (Spearman Rho $=0.71, P<0.001$ ), and fatigue (Spearman Rho = $0.35, P<0.001)$ were all correlated with the total score of the SCOPA-PS questionnaire. Figure 1 illustrates the scatter plot between the total SCOPA-PS score and HADS scores of anxiety and depression.

\section{Discussion}

Until now, SCOPA-PS has been translated into LatinAmerican and few European languages [15, 16], yet no Persian-translated version of SCOPA-PS is available in Iran and the present study is the first attempt in validating this version of SCOPA-PS. Indeed, this effort is of utmost importance 


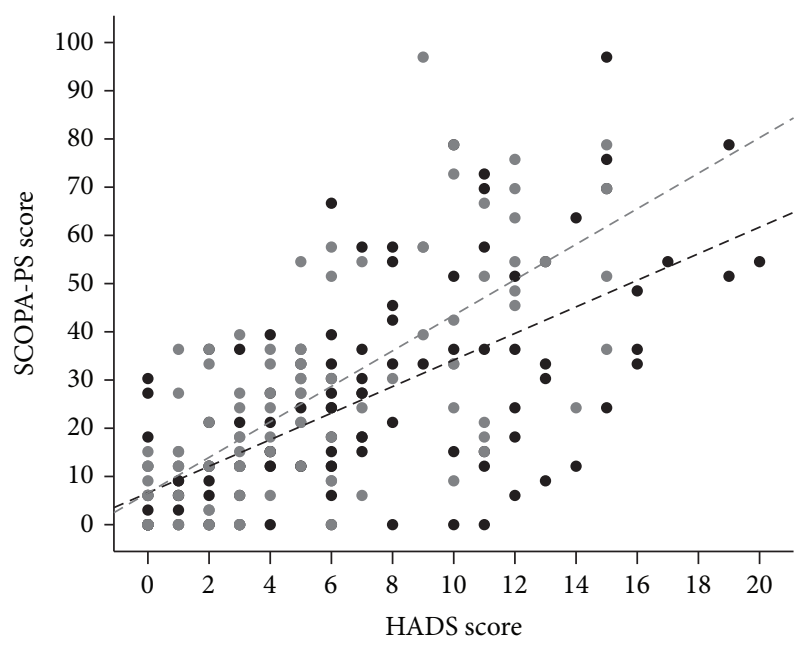

FIgURE 1: Scatter plot of the correlation between total score of SCOPA-PS and HADS questionnaires for anxiety (black circles; Spearman Rho $=0.642, P<0.001$ ) and depression (gray circles, Spearman Rho $=0.715, P<0.001)$ in Iranian Parkinson's disease patients $(n=110)$.

TABLE 4: Spearman correlation for convergent validity of the SCOPA-PS questionnaire regarding other measured scales and variables in Iranian Parkinson's disease patients $(n=110)$.

\begin{tabular}{lc}
\hline Scale/variable & Spearman Rho \\
\hline Age & 0.07 \\
Duration of disease & 0.17 \\
Hoehn and Yahr stage & $0.34^{*}$ \\
Schwab and England ADL scale (\%) & $-0.55^{*}$ \\
PDQ-39 (quality of life) & \\
Mobility & $0.72^{*}$ \\
Activities of daily living (ADL) & $0.60^{*}$ \\
Emotional well-being & $0.63^{*}$ \\
Stigma & $0.46^{*}$ \\
Social support & $0.43^{*}$ \\
Cognitive impairment (cognition) & $0.54^{*}$ \\
Communication & $0.54^{*}$ \\
Bodily discomfort & $0.44^{*}$ \\
Total & $\mathbf{0 . 8 2 ^ { * }}$ \\
HADS & \\
Anxiety & $0.64^{*}$ \\
Depression & $0.72^{*}$ \\
FSS (fatigue) & $0.36^{*}$ \\
\hline
\end{tabular}

${ }^{*}$ Statistical significant correlation at the level of $P<0.001$.

to validate an instrument to measure psychosocial burden of $\mathrm{PD}$ in patient population.

The internal consistency of SCOPA-PS was acceptable (Cronbach's alpha $=0.87$ ) in our study and was even higher than the original study and the Brazilian version (Cronbach's alpha $=0.83)[14,16]$ where the intraclass correlation was also high. Results of similar studies on validity and reliability of the SCOPA-PS in different languages are summarized in Table 5. Originally, Marinus et al. developed the SCOPAPS to evaluate psychosocial functioning in PD patients. One hundred and seventeen PD patients filled the Dutch version of the SCOPA-PS in stage 1 to 5 of disease severity according to Hoehn and Yahr. The result revealed that the Dutch version of SCOPA-PS had good internal consistency (Cronbach's alpha $=0.83)[14]$.

In item-specific analysis, the highest correlation score (0.76) referred to item 7 on "asking for help" which indicates the highest internal consistency with the total SCOPA-PS score. Even though all coefficients were above 0.4 as the limit for item consistency, the lowest coefficient $(0.55)$ was observed for item 5 on "sexuality." Moreover, deletion of none of the questions would increase the total Cronbach's alpha coefficient above the formerly calculated one, which means that all of the questions are suitable for the internal consistency. However, cultural barriers about sexual aspects and taboo considerations may cause the relatively low internal consistency of item 5 on psychosocial problems with "sexuality." In line with our findings, the lowest consistency coefficient in both the original Dutch [14] and the German versions [15] of the questionnaire was also reported for item 5 on "sexuality", whereas in the Brazilian [16] and Spanish versions [8] item 10 on "feeling ashamed of disease" showed the lowest consistency. Interestingly, the coefficient for the "sexuality" item was found to be higher in our study compared to the reports from the Netherlands [14] and Germany [15], while the opposite might have been expected according to the sociocultural characteristics. This may highlight the evolutionary transition of current Iranian society, showing less taboo talking about sexual issues especially in the group of patients with chronic disabilities. At the same time, it could also indicate the importance of sexual problems in the functioning of Parkinsonian patients that makes them precisely answer the question on sexuality. This finding was actually against our expectations prior to data collection to encounter problems in the validity and reliability of the "sexuality" item.

According to our results, patients with higher Hoehn and Yahr stage and lower Schwab and England ADL scale had higher SCOPA-PS score showing the fact that patients with more severe PD have more problems with their psychosocial functioning. Different psychiatric features including anxiety $(r=0.64)$, depression $(r=0.72)$, fatigue $(r=0.36)$, and all of the subscales and entire HRQoL measured by the PDQ-39 were also correlated with the total score of SCOPAPS. These findings not only further confirm the validity of the Persian version of the SCOPA-PS questionnaire, but also show the important relationships between different symptoms (both motor and nonmotor) of PD and psychosocial malfunctioning in patients. Previously, the Brazilian study of SCOPA-PS also showed significant correlation between total score of SCOPA-PS and anxiety $(r=0.51)$, depression $(r=0.49)$, and Hoehn and Yahr stage $(r=0.38)$ [16]. Likewise, in the original study that developed the SCOPAPS with a sample size of 338 PD patients, total score of the questionnaire was significantly correlated with PDQ-39 score $(r=0.82)$, anxiety $(r=0.62)$, and depression $(r=0.61)$ [14]. 
TABLE 5: Validity, reliability, and psychometric properties of the SCOPA-PS in different languages/cultures (two different coefficients are reported for some indices to show the lowest and highest item-specific calculated reliability).

\begin{tabular}{|c|c|c|c|c|c|c|c|}
\hline \multirow[b]{2}{*}{ Authors } & \multirow[b]{2}{*}{ Year } & \multirow[b]{2}{*}{ Country/language } & \multicolumn{2}{|c|}{ Reliability } & \multicolumn{3}{|c|}{ Validity } \\
\hline & & & $\begin{array}{c}\text { Internal } \\
\text { consistency } \\
(\text { Spearman R) }\end{array}$ & $\begin{array}{c}\text { Internal } \\
\text { consistency } \\
\text { (Cronbach's } \\
\text { alpha) } \\
\end{array}$ & $\begin{array}{c}\text { Correlation } \\
\text { coefficient with } \\
\text { HADS (anxiety) }\end{array}$ & $\begin{array}{c}\text { Correlation } \\
\text { coefficient with } \\
\text { HADS } \\
\text { (depression) } \\
\end{array}$ & $\begin{array}{c}\text { Correlation } \\
\text { coefficient with } \\
\text { HRQoL } \\
(\text { PDQ-39) } \\
\end{array}$ \\
\hline Marinus et al. [14] & 2003 & $\begin{array}{c}\text { The } \\
\text { Netherlands/Dutch }\end{array}$ & $\begin{array}{l}0.24 \text { (item 5) } \\
0.67 \text { (item 7) } \\
\end{array}$ & 0.83 & 0.61 & 0.62 & 0.82 \\
\hline $\begin{array}{l}\text { Carod-Artal et al. } \\
{[16]}\end{array}$ & 2006 & $\begin{array}{l}\text { Brazil/Brazilian } \\
\text { and Portuguese }\end{array}$ & $\begin{array}{c}0.43 \text { (item } 10) \\
0.73 \text { (item 9) } \\
\end{array}$ & 0.84 & 0.50 & 0.47 & 0.73 \\
\hline $\begin{array}{l}\text { Virués-Ortega et } \\
\text { al. [8] }\end{array}$ & 2009 & $\begin{array}{c}\text { Argentina, } \\
\text { Paraguay, Ecuador, } \\
\text { Brazil/Spanish and } \\
\text { Portuguese }\end{array}$ & $\begin{array}{c}0.43(\text { item } 10) \\
0.71(\text { item } 9)\end{array}$ & 0.87 & 0.62 & 0.61 & 0.82 \\
\hline $\begin{array}{l}\text { Knudsen et al. } \\
{[15]}\end{array}$ & 2007 & Germany/German & $\begin{array}{l}0.35 \text { (item 5) } \\
0.77 \text { (item 6) }\end{array}$ & 0.90 & 0.76 & 0.76 & 0.86 \\
\hline Current study & 2013 & Iran/Persian & $\begin{array}{l}0.55 \text { (item 5) } \\
0.76 \text { (item 7) }\end{array}$ & 0.87 & 0.64 & 0.71 & 0.82 \\
\hline
\end{tabular}

* The reported correlation coefficients refer to the total score of the PDQ-39 questionnaire.

As one strength point, we used several scales and questionnaires to consider PD severity and different motor, nonmotor, and psychiatric features in relation with the psychosocial functioning of PD patients. Therefore, it was possible to evaluate different relationships and interactions in cross-cultural validation of the Persian version of SCOPA-PS. However, this study was designed as a cross-sectional study, which made it impossible to evaluate test-retest reliability, and any interpretation on causal relationships between PDrelated symptoms and psychosocial functioning could not be made. Another limitation is based on the fact that the present study was performed in an outpatient clinic that mostly included mild-to-moderate PD patients, which makes it difficult to generalize our findings to the end-stage and hospitalized patients.

\section{Conclusions}

In conclusion, our study demonstrated that the Persian version of SCOPA-PS is a reliable and valid scale to measure psychosocial functioning in IPD patients with different sex, age-group, and educational level. This cross-culturally validated version of SCOPA-PS has high reliability for individual assessment. Opposite to our prestudy assumptions, Iranian PD patients precisely answered the SCOPA-PS questionnaire and less cross-cultural validation was needed to adopt the items of this questionnaire with the sociodemographic contexts of our society.

During the process of convergent validity, PD severity scales, depression, anxiety, fatigue, and different domains of HRQoL were all associated with psychosocial functioning measured by the SCOPA-PS. Comparing with results from other similar studies, the item-specific analysis confirmed the importance of cross-cultural validation for psychological scales; for instance, in some cultures, the answers to questions about sexuality are less valid. In total, our investigation recommends the Persian version of the SCOPA-PS questionnaire as a valid and reliable tool to measure psychosocial functioning in PD patients, which could be used in future projects.

\section{Conflict of Interests}

The authors declare that they have no conflict of interests regarding the publication of this paper.

\section{Acknowledgments}

The authors have neither financial disclosures nor conflict of interests to declare in relation to the content of this paper. The authors are grateful to the colleagues who contributed in data collection from the movement disorder clinic: Mahdyeh Shafiei Sabet, Dena Khaefpanah, Nader Naderi, Arash Rahmani, Ms. Mahmoudi, and Poorang Bassir who helped us in the preparation of the questionnaire. The authors also thank all patients and caregivers for their collaboration to collect the data.

\section{References}

[1] M. C. de Rijk, L. J. Launer, K. Berger et al., "Prevalence of Parkinson's disease in Europe: a collaborative study of population-based cohorts," Neurology, vol. 54, no. 11, supplement, pp. S21-S23, 2000.

[2] C. J. L. Murray and A. D. Lopez, "Evidence-based health policy: lessons from the global burden of disease study," Science, vol. 274, no. 5288, pp. 740-743, 1996.

[3] J. Simpson, K. Haines, G. Lekwuwa, J. Wardle, and T. Crawford, "Social support and psychological outcome in people with 
Parkinson's disease: evidence for a specific pattern of associations," British Journal of Clinical Psychology, vol. 45, no. 4, pp. 585-590, 2006.

[4] K. R. Chaudhuri, L. Yates, and P. Martinez-Martin, "The nonmotor symptom complex of Parkinson's disease: a comprehensive assessment is essential," Current Neurology and Neuroscience Reports, vol. 5, no. 4, pp. 275-283, 2005.

[5] A. Schrag, M. Jahanshahi, and N. Quinn, "What contributes to quality of life in patients with Parkinson's disease?" Journal of Neurology Neurosurgery and Psychiatry, vol. 69, no. 3, pp. 308312, 2000.

[6] P. Martinez-Martin and W. C. Koller, Quality of Life in Parkinson's disease, Masson, Barcelona, Spain, 1999.

[7] B. S. Singhal, "Neurology in developing countries: a population perspective," Archives of Neurology, vol. 55, no. 7, pp. 1019-1021, 1998.

[8] J. Virués-Ortega, F. J. Carod-Artal, M. Serrano-Dueñas et al., "Cross-cultural validation of the Scales for Outcomes in Parkinson's Disease-Psychosocial questionnaire (SCOPA-PS) in four Latin American countries," Value in Health, vol. 12, no. 2, pp. 385-391, 2009.

[9] P. Martínez Martín, B. Frades, F. J. Jiménez Jiménez et al., “The PDQ-39 Spanish version: reliability and correlation with the short-form health survey (SF-36)," Neurologia, vol. 14, no. 4, pp. 159-163, 1999.

[10] D. M. Bushnell and M. L. Martin, "Quality of life and Parkinson's disease: translation and validation of the US Parkinson's Disease Questionnaire (PDQ-39)," Quality of Life Research, vol. 8, no. 4, pp. 345-350, 1999.

[11] M. Serrano-Dueñas, P. Martínez-Martín, and V. VacaBaquero, "Validation and cross-cultural adjustment of PDQL-questionnaire, Spanish version (Ecuador) (PDQL-EV)," Parkinsonism and Related Disorders, vol. 10, no. 7, pp. 433-437, 2004.

[12] P. Martínez-Martín, M. Serrano-Dueñas, and V. Vaca-Baquero, "Psychometric characteristics of the Parkinson's disease questionnaire (PDQ-39): ecuadorian version," Parkinsonism and Related Disorders, vol. 11, no. 5, pp. 297-304, 2005.

[13] K.-L. Tsang, I. Chi, S.-L. Ho, V. W. Lou, T. M. C. Lee, and L.W. Chu, "Translation and validation of the standard Chinese version of PDQ-39: a quality-of-life measure for patients with Parkinson's disease," Movement Disorders, vol. 17, no. 5, pp. 1036-1040, 2002.

[14] J. Marinus, M. Visser, P. Martínez-Martín, J. J. van Hilten, and A. M. Stiggelbout, "A short psychosocial questionnaire for patients with Parkinson's disease: the SCOPA-PS," Journal of Clinical Epidemiology, vol. 56, no. 1, pp. 61-67, 2003.

[15] K. Knudsen, P. Martinez-Martin, and C. Deuschl, "Evaluation of the reliability and validity of the German version of a questionnaire on the psychosocial impairment of patients with Parkinson's disease," Aktuelle Neurologie, vol. 34, no. 5, pp. 267271, 2007.

[16] F. J. Carod-Artal, P. Martinez-Martin, and A. P. Vargas, "Independent validation of SCOPA-psychosocial and metric properties of the PDQ-39 Brazilian version," Movement Disorders, vol. 22, no. 1, pp. 91-98, 2007.

[17] A. J. Hughes, S. E. Daniel, L. Kilford, and A. J. Lees, "Accuracy of clinical diagnosis of idiopathic Parkinson's disease: a clinicopathological study of 100 cases," Journal of Neurology Neurosurgery and Psychiatry, vol. 55, no. 3, pp. 181-184, 1992.
[18] M. F. Folstein, S. E. Folstein, and P. R. McHugh, “Mini mental state': a practical method for grading the cognitive state of patients for the clinician," Journal of Psychiatric Research, vol. 12, no. 3, pp. 189-198, 1975.

[19] M. M. Hoehn and M. D. Yahr, "Parkinsonism: onset, progression and mortality," Neurology, vol. 17, no. 5, pp. 427-442, 1967.

[20] S. M. Fereshtehnejad, H. Hadizadeh, F. Farhadi, G. A. Shahidi, A. Delbari, and J. Lokk, "Reliability and validity of the persian version of the fatigue severity scale in idiopathic Parkinson's disease patients," Parkinsons Dis, vol. 2013, Article ID 935429, 7 pages, 2013.

[21] L. B. Krupp, N. G. LaRocca, J. Muir-Nash, and A. D. Steinberg, "The fatigue severity scale: application to patients with multiple sclerosis and systemic lupus erythematosus," Archives of Neurology, vol. 46, no. 10, pp. 1121-1123, 1989.

[22] A. Montazeri, M. Vahdaninia, M. Ebrahimi, and S. Jarvandi, "The Hospital Anxiety and Depression Scale (HADS): translation and validation study of the Iranian version," Health and Quality of Life Outcomes, vol. 1, article 14, 2003.

[23] A. S. Zigmond and R. P. Snaith, "The hospital anxiety and depression scale," Acta Psychiatrica Scandinavica, vol. 67, no. 6, pp. 361-370, 1983.

[24] V. Peto, C. Jenkinson, and R. Fitzpatrick, "PDQ-39: a review of the development, validation and application of a Parkinson's disease quality of life questionnaire and its associated measures," Journal of Neurology, vol. 245, 1, pp. S10-S14, 1998.

[25] M. Nojomi, Z. Mostafavian, G. A. Shahidi, and C. Jenkinson, "Quality of life in patients with Parkinson's disease: translation and psychometric evaluation of the Iranian version of PDQ-39," Journal of Research in Medical Sciences, vol. 15, no. 2, pp. 63-69, 2010.

[26] P. M. Fayers, Quality of Life: Assessment, Analysis and Interpretation, John Wiley and Sons, New York, NY, USA, 2000. 


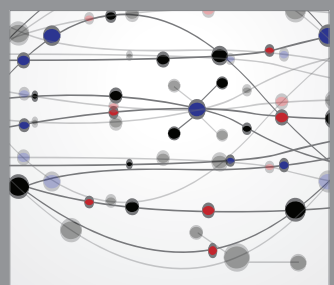

The Scientific World Journal
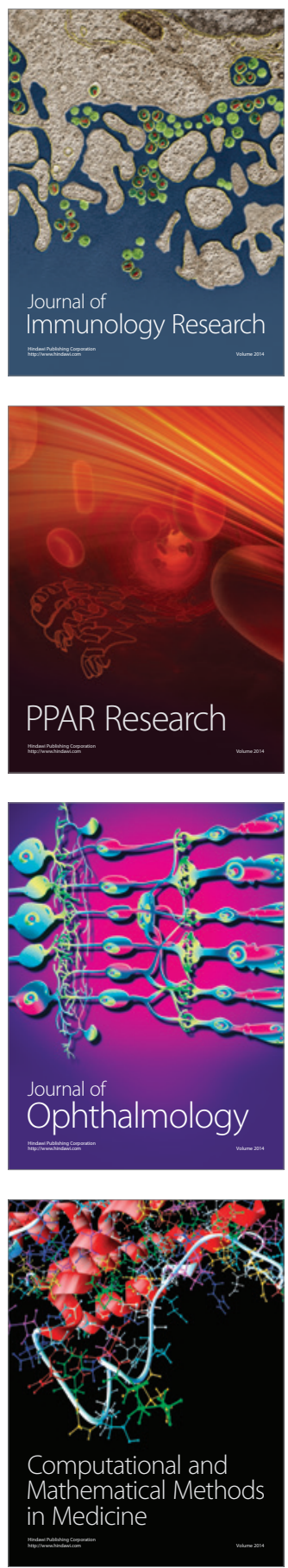

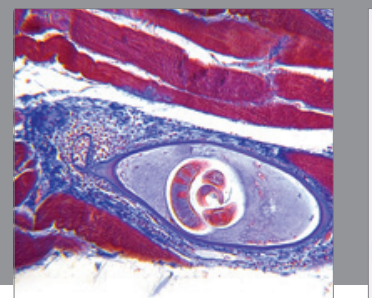

Gastroenterology

Research and Practice
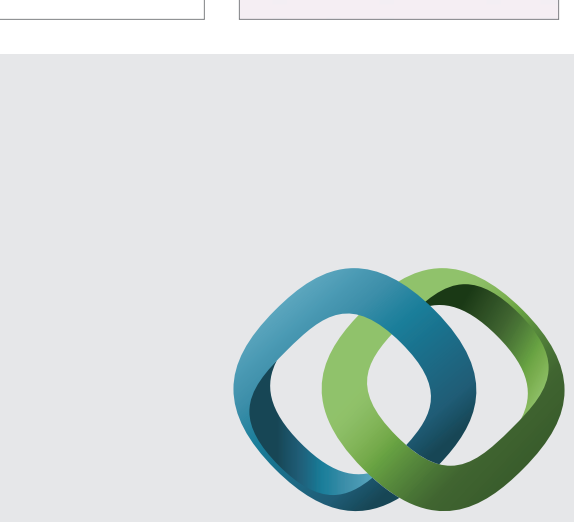

\section{Hindawi}

Submit your manuscripts at

http://www.hindawi.com
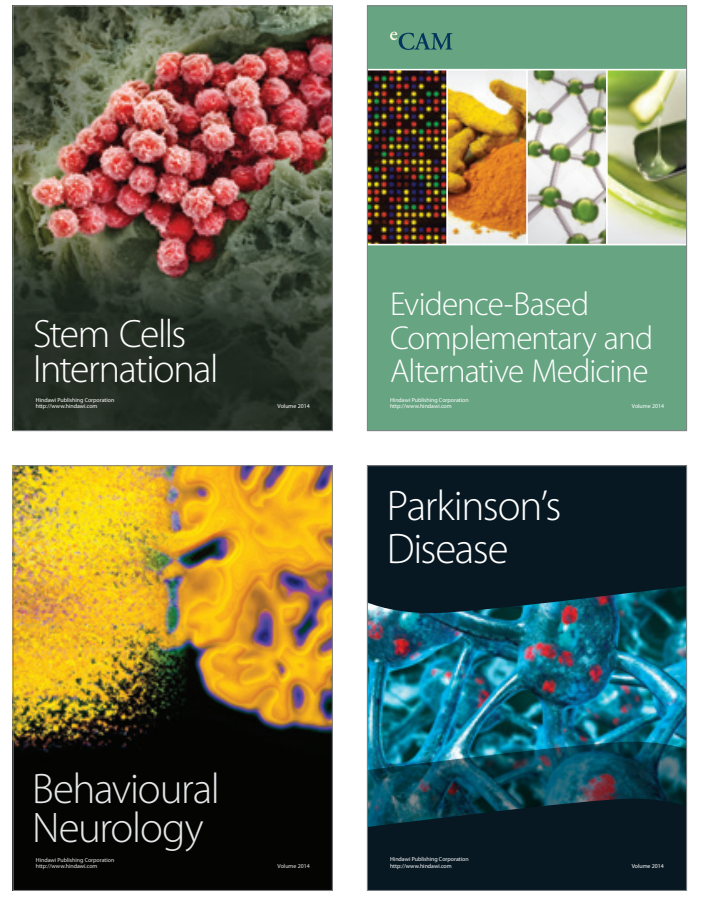
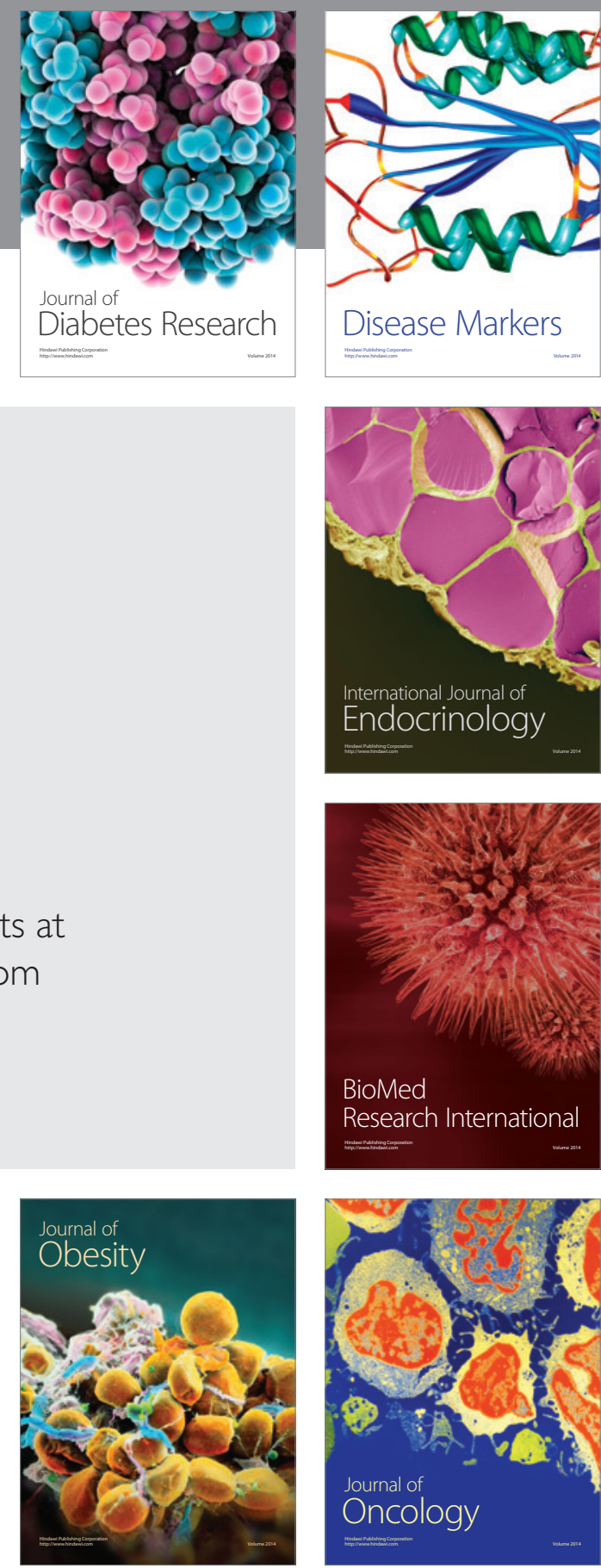

Disease Markers
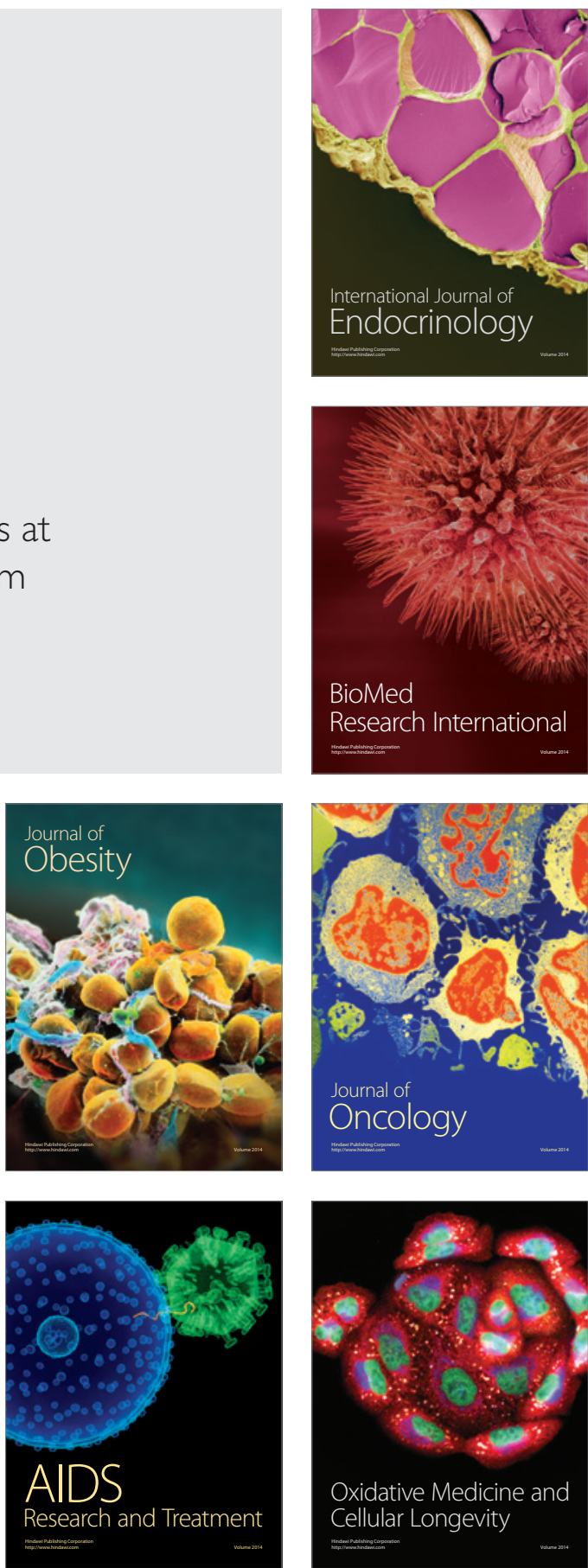